\title{
Telemedicine: Bridging the Gap between Refugee Health and Health Services Accessibility in Hamilton, Ontario
}

\author{
ANTHONY ROBERT SANDRE AND K. BRUCE NEWBOLD
}

\begin{abstract}
Refugees face considerable challenges upon seeking asylum in Canada, and accessing health care services remains a prominent issue. Recurrent themes in the literature out lining barriers to health-services accessibility include geographic, economic, and cultural barriers. Drawing on the experiences of service providers in Hamilton, Ontario, we explored the efficacy of telemedicine services in bridging the gap between refugee health and health-services accessibility. Research methodology included structured interviews with clinicians who provide health-care services to refugees, complemented by a scoping literature review. The results of this exploratory study demonstrate the efficacy of telemedicine in encouraging dialogue and policy change in the greater health-care setting, and its potential to increase access to specialist health-care services.
\end{abstract}

\section{Résumé}

Les réfugiés doivent faire face à des défis considérables lors $d u$ processus de demande d'asile au Canada, et l'accès aux services de santé demeure un enjeu important. Parmi les préoccupations qui reviennent fréquemment dans la documentation portant sur l'accessibilité aux services de santé sont les obstacles de nature géographique, économique, et culturelle. En nous basant sur l'expérience vécue des fournisseurs de service établis à Hamilton, en Ontario, nous étudions l'efficacité des services de télémédecine à combler l'écart entre les besoins en matière de santé des réfugiés et l'accessibilité aux services de santé. La méthodologie de recherche comportait des entrevues structurées avec le personnel traitant chargé de fournir des services de santé aux réfugiés, accompagnée d'une revue exploratoire de la documentation sur le sujet. Les résultats de cette étude exploratoire ont démontré l'efficacité de la télémédecine à stimuler le dialogue et le changement en matière de politique dans le contexte général des services de santé, ainsi que sa capacité à accroître l'accès aux services de santé spécialisés.

\section{Introduction}

7 he social, ${ }^{1}$ health, ${ }^{2}$ and medical ${ }^{3}$ needs of refugee populations are unique. Refugees are more likely to have experienced combat and domestic violence; 4 political instability and political warfare; death of family and friends; and culture shock. ${ }^{5}$ The combination of these adverse events before, during, or after migration frequently manifest as physical and mental health issues, predominantly post-traumatic stress disorder, generalized anxiety disorder, and depression. ${ }^{6}$

Several international systematic reviews have outlined general barriers to accessing health-care services across all vulnerable populations, i.e., immigrants, refugees, and asylum-seekers. Carrasco, Gillespie, and Goodluck outline the challenges for immigrants in accessing primary-care services in Canada, where primary care is considered to be the first point of contact with medical services or the health-care system, usually mediated by a family physician. ${ }^{7}$ Medical practitioners who were unable to address the needs of immigrants, that is, unwilling to accommodate the 
culturally sensitive demands of the patient or unwilling to participate in culturally sensitive training, deterred patients from accessing services. ${ }^{8}$ Joshi et al. draw on similar principles in emphasizing the need to provide identical health care to refugees and other members of the general public. ${ }^{9}$ The authors call for regular physician-patient communication in the provision of primary-care services in order to improve access to and quality of health-care services. ${ }^{10}$ Hadgkiss and Renzaho explored the utilization of healthcare services for asylum-seekers in Australia. Six general themes were identified: affordability, including transportation and prescription medication costs; poor health literacy and understanding of the health system; perceived effectiveness and quality of health services; medical mistrust; discrimination and health professionals' attitudes, or witnessed substandard treatment of patients; and linguistic and cultural factors. ${ }^{11}$

Accessibility to health care in Canada is guided by the 1984 Canada Health Act, with the over-arching objective to ensure that all medically necessary services will be provided free of charge, with the implication of unimpeded access to health-care services for all. ${ }^{12}$ In considering these principles and the fluidity of the Canadian refugee population, the Government of Canada enacted the Interim Federal Health Program (IFHP). ${ }^{13}$ Currently, and following major restructuring in 2012, 2014, and again in 2016, the IFHP provides health coverage to protected persons (resettled refugees), government-assisted refugees, refugee claimants, and other specified groups. ${ }^{14}$ The IFHP is a temporary health insurance program for non-Canadian citizens, and six types of coverage are offered on the basis of immigration status of the individual. ${ }^{15}$ Recurrent barriers, including a limited number of subsidized health services, call for a solution to health services accessibility between clinician and patient. ${ }^{16}$

Telemedicine or telehealth, which refers to the provision of health-care services using specialized technology, ${ }^{17}$ spans consultation, ${ }^{18}$ referral, diagnosis, treatment, and followup. ${ }^{19}$ Notably, over an array of medical specialties, telemedicine has been shown to increase satisfaction for patient and health-care provider; 20 increase services access for vulnerable and distant populations; ${ }^{21}$ and improve linguistic and cultural appropriateness of care. ${ }^{22}$ Within Ontario, existing and well-known telemedicine services include Telehealth Ontario $^{23}$ and the Ontario Telemedicine Network (отN). ${ }^{24}$ Telehealth Ontario provides patients with 24/7 phone access to a registered nurse who may assist in symptom management and/or booking appointments with other health-care providers; all services are free for citizens of Ontario. ${ }^{25}$ The OTN was created to link patients in rural and remote settings with health-care providers across the province using two-way videoconferencing. ${ }^{26}$ Although telemedicine is classified as an uninsured service, physicians offering their services through OTN will submit their bills for consultation to the Ontario Health Insurance Program (OHIP). ${ }^{27}$ In light of the IFHP and its divide from OHIP, the medical-legal landscape in Canada poses immediate barriers to the care of refugee patients via technology, e.g., Telehealth and oTn.

While several authors have explored the efficacy of telemedicine in addressing the health intricacies of vulnerable and underserved populations, few authors have explicitly focused on its applicability to refugee populations. Herein, we propose telemedicine as a means to bridge the gap between refugee-health and health-services accessibility for refugee populations. The objective of this study is to explore the efficacy of telemedicine for remediating healthservices accessibility for refugees, with special attention paid to accessing specialist care. In order to understand the relationship between telemedicine and health-services accessibility for refugee populations, research methodology included structured interviews with clinicians who provide health care to refugees in Hamilton, complemented by a scoping literature review focused on the implementation and delivery of telemedicine services to vulnerable and/or underserved populations. This study will also contribute to the existing literature concerned with barriers to accessing health-care services for refugee populations. The authors hope that this study will serve as a dialogue piece surrounding health inequity for refugees, and will encourage clinicians to implement telemedicine services in their practice.

\section{Methods}

Two research methods were used to explore the efficacy of telemedicine to bridge the gap between refugee health (and its intricacies) and health-services accessibility. Data obtained from structured interviews with health-care professionals who provide health care to refugees in Hamilton were complemented by a scoping literature review.

Qualitative research methods provide an understanding of personal truths, and five physicians and one nurse practitioner were interviewed in 2015 and 2016. Research ethics board clearance was obtained from the Hamilton Integrated Research Ethics Board of McMaster University. Non-probability, purposive sampling of health-care professionals was performed to obtain the study sample. Three family physicians, one internal medicine subspecialist (subspecializations: medical microbiology and infectious diseases), one pediatrician, and one nurse practitioner were interviewed. In an effort to improve the credibility of results, we sampled a unique and interdisciplinary team, including one family physician who was a former refugee. Participants were contacted by electronic mail and were identified using physician referral and/or place of employment. Participants 
were fully informed of the research objective, study design, results reporting, confidentiality of information, and the intended use of results. Participants remain anonymous, save professional qualifications. One-on-one interviews were recorded, and each respondent was asked six questions about the implementation and delivery of telemedicine services when serving refugee populations.

Open coding methods were utilized to interpret the collected data, and underlying themes were identified, labelled, and categorized. ${ }^{28}$ In order to elucidate an in-depth understanding of the opinions of the health-care providers and their experiences in working with refugees, analysis followed grounded theory. Grounded theory methodology necessitates constant comparison between accounts, i.e., health-care provider perceptions, and through repeated and systematic assessment, the author is able to generate social truths grounded in empirical data. ${ }^{29}$

A scoping literature review was conducted in order to complement interview data and to perform a rapid and encompassing assessment of the current and existing literature within the scope of this research article. In comparison to systematic review counterparts, scoping literature reviews do not include a formal quality assessment of the literature; rather, a methodical presentation of the literature, including categorization and reporting of data using specified search criteria and databases. ${ }^{30}$ Arksey and O'Malley state that there are five essential steps of any scoping literature review, including identifying a research question, finding and selecting relevant studies, charting data, and reporting said data methodically. ${ }^{31}$ Further, they state that the author may choose to consult relevant stakeholders to provide direct insight onto the literature. ${ }^{32}$

Electronic databases of MEDLINE, Embase, and PubMed were searched in January 2016 for the terms emigrants and immigrants or refugees or transients and migrants or vulnerable populations AND telemedicine; and one hundred and twenty-six references were obtained. A breadth of search terms were included for two reasons: first, because there was a scarcity of literature focused explicitly on telemedicine and refugee health; and second, while this study is focused on refugee health, the authors believe that most, if not all, core social and health concepts found using these search terms can be applied across vulnerable populations (including refugees). Seventy-four references were obtained after redundancies were eliminated. Next, only peer-reviewed, English articles published since 2000 and accessible by the McMaster University electronic database were included. Any articles focused on the delivery of dental-care services, rather than medical-care services, were eliminated, reducing the number of references to fifty-one. Finally, in order to maintain the integrity of the research objective, any articles concerning physician migration, incarcerated individuals, or smoking cessation were excluded. Articles were also required to focus on the delivery of a telemedicine service (rather than intervention design). The remaining twentyseven references were reviewed and determined to satisfy the inclusion criteria. The authors note that any research papers referenced in the introduction section have been included only to explain core concepts. All research papers referenced in subsequent sections are the result of the scoping literature review.

\section{Results}

\section{Structured Interviews}

Stakeholder perceptions obtained during structured interviews revealed three themes concerning the implementation and delivery of telemedicine when serving refugee populations: (1) model of care and understanding of health, (2) perceived benefits, and (3) perceived challenges associated with the implementation of telemedicine services in the health-care setting. Verbatim quotes have been included to demonstrate participant perceptions.

\section{Model of Care and Understanding of Health}

In keeping with the diverse perspectives offered by the participants in this study, all health-care providers were acutely aware of the unique health needs of refugee populations, both upon arrival and while residing in Canada. There was unanimous agreement amongst health-care providers that the current model of care and understanding of health continues to be the greatest barrier faced by refugee patients when attempting to access health-care services. This biomedical model surpasses simple differences in language, a frequently cited barrier, and exacerbates differences in the expectation and understanding of chronicity of disease; differences (or similarities) amongst health-care provider and patient expectations; in-depth understanding of foreign culture; and, of course, frustration in navigating refugeehealth legislation.

Language, visualized as the "tip of the iceberg" in the model of care and understanding of health, spans appointments, communicating with health-care professionals, comprehending reading materials, and understanding diagnosis and/or prognosis. Among participants, there was consensus that language, an extension of ethnic diversity, challenges health-care providers and patients alike:

Despite advancement, I believe that language remains the biggest barrier, in terms of making an appointment or having an appointment being made for you, seeking specialist opinions or locating health-care services. The patient may also have difficulty accessing and understanding such information if it is located in a central place, e.g., a website. (Participant 1) 
One barrier is language. This can be a challenge if there is or is not an interpreter-this can make [patient care] very challenging. It may be that the language may interfere with referring to the specialist [or performing] investigations as well as a breakdown in communication. (Participant 3)

While language serves as a concrete barrier to patients and health-care providers, this concept is further enveloped in a network of cultural and human understanding, i.e., the model of care and understanding of health offered by the health-care provider. By simply providing the patient with an interpreter, the health-care provider does not break down this barrier; rather, he or she exacerbates it:

In my experience, people spend a lot of time to talk diabetes-but from a framework that the client isn't engaged with. So who cares? They have an interpreter, and they have a diabetes educator, and they're talking diabetes education, and they have the time, but the model doesn't fit in [the patient's] mind, you know, about chronic disease, or about lifestyle management, or about foods that are best, you know, things like that-so the communication still hasn't taken place ... The biggest barrier to providing care to new patients is the biomedical model that we work under; because, perhaps, it's contrary, a bit to what they've experienced in the past ... There, perhaps, might not be an understanding of chronicity of disease, the use of medication, diagnosis, and what we mean .. especially by mental health. People come with huge histories of trauma, but then their symptoms are somatic, and because we are very biomedical, we investigate the symptoms without thinking about it ... And, it's not, perhaps, that person's way of expressing distress. (Participant 4)

Obviously language and the whole cultural piece; people have different expectations of the health-care systems, depending where they're from. (Participant 6)

As a former refugee, the next participant, a family physician, provides an in-depth understanding of the issues a refugee may face when adjusting to a new model of care and understanding of health:

I have, and still do, work with refugees. I was a refugee myself ... So when I became a doctor, of course, my priority was immigrants and refugees ... They are a challenge, the refugees, because their issues are not single. They are not only medical. It's a whole social circumstance. (Participant 5)

Variability in the delivery of health-care services between the origin and settled countries challenges the health-care provider and patient. Certain physicians may be unwilling to work with refugee patients:
There is a certain degree of stigma associated with being a refugee. This makes it hard just to get access to health care, because there is a perception that this is a more difficult population to deal with for a number of different reasons: cultural understanding, language barriers, those types of issues. (Participant 2)

The current model of care and understanding of health also necessitates a mention of the issues surrounding compensation for the health-care provider and frustration when navigating refugee-health legislation. Each participant explicitly voiced this concern. Financial compensation, an extension of health policy, has challenged participants:

There's the financial aspect, a barrier imposed by policy, number one, and the health-care system, number two ... [so] physicians find it difficult to navigate the compensation program for refugees since it is a very complicated system-oftentimes physicians just won't see refugee patients. (Participant 2)

A big component, obviously in the last few years, is IFHP cuts. The actual confusion at the health-care-provider level ... essentially people who used to perhaps figure it out once in awhile are no longer willing to do that. More [refugees] are just turned away because it's too confusing or they are not covered. (Participant 1)

The accessibility of health services extends into exploring solutions to these barriers. While participants did mention telemedicine as a potential solution, respondents also described a non-electronic intervention-a communitybased partnered approach. The scope of this concept is twofold: first, the evolution of a specialized facility to serve refugee populations, and second, building a professional relationship with community members who are of a similar religious and/or ethnic background of the patient:

The best way to serve this population is through a community health centre. (Participant 5)

The most success we've had is a community-based partnered approach ... Newcomers are accessing their services for housing, school, employment, and everything else ... and then receiving health care in the same location in collaboration with settlement workers. So them being able to work alongside the [settlement workers] for questions the family may have around school, integration, etc. [As physicians], we work closely with [the settlement workers] to get initial referrals for newcomers-they can direct them to a clinic where they'll be seen regardless of coverage and where we have access to translators. (Participant 1)

From a primary-care point of view, the general response has been the evolution of a specialized primary-care clinic that will

(C) Anthony Robert Sandre and K. Bruce Newbold, 2016. This open-access work is licensed under a Creative Commons AttributionNonCommercial 4.0 International License, which permits use, reproduction and distribution in any medium for non-commercial purposes, provided the original author(s) are credited and the original publication in Refuge: Canada's Journal on Refugees is cited. 
see refugee patients and is aimed to get past the financial burden aspect of it ... there is at least a presumably higher cultural understanding in that clinic and there is language services available for translation. (Participant 2)

For language support, from my perspective, the ideal is to have an in-person translator who knows the family in a professional context. So not a community member that [the patient feels] wouldn't be confidential, which can be tricky in some of the less common languages. (Participant 1)

The advantage of establishing a professional relationship with community members, who can assist the health-care professional in providing a higher standard of care, is demonstrated in the following quotation:

The time is spent with interpreters to talk about, say, post-traumatic stress, but when we're talking from the same frameworkand I'm not talking where we have to go figure out what [ethnicity] they are and go change the language-it's more like having more of a human understanding of what the thinking is back and forth. (Participant 4)

\section{Perceived Benefits Associated with Implementation of Telemedicine}

With the exception of telephone translation services, all participants had limited experience with the implementation and facilitation of telemedicine; however, all broadly spoke of the perceived benefits associated with offering such services:

I've seen telehealth work really well when a primary-care provider, such as a nurse, nurse practitioner, or family physician, is with the patient on one side while communicating with a specialist on the other side. (Participant 1)

I did a physician search for doctors who speak Somali, and only one came up in the area, and I already work with him. He's very, very busy, you know. So what if there were ten, twenty, who knows how many in Toronto, that would be willing-particularly if there was compensation - to be able to consult, and then we could just ... manage that way. Yeah, that would be superb. (Participant 4)

I'm thinking, envisioning that ... if I can't find a psychiatrist in Hamilton to do an assessment ... Let's say there was a psychiatrist, Farsi-speaking or Afghani-speaking in Toronto, and willing to do the assessment through telemedicine from my own office, I would set up the patient, have them do the interview, and everything .. That would be a proper assessment, because not everything needs the patient to be physically touched. (Participant 5)
I did call the on-call pediatrician, but it would have been great if they could have seen it. (Participant 6)

Another aspect is getting patients access to specialist care. This works best if the primary-careprimary care clinic, with the patient alongside, can communicate to the specialist or his or her office using telehealth. (Participant 2)

Further, telemedicine increases the human resource capacity (and referral network) available to the health-care provider, who can then provide a higher standard of care to the patient:

There's actually an application for a nurse practitioner to do telemedicine NP for rural, right? ... Usually it's been a physician and now they're bringing nurse practitioners into this model. And it might be part of the solution, I was thinking around all this, to our issues around human resources, because our clients have multiple needs-sometimes, not all the time, some of them are just really, really healthy and adapt and there is no problems at all-but sometimes they have a lot of needs and you have to bring them a lot of specialists, and we do that by bringing them physically on site. But would it also be possible to have more nurses or something, or some other means of accessing specialists, from telemedicine?

I could see it as a way of expanding the human resource or the clinical capacity for not just refugees, but all marginalized populations. (Participant 4)

Finally, visualizing human interaction over an electronic medium allows the clinician to observe physical nuances:

But to be able to catch the nuances of symptoms in one's own language, instead of even through a medically trained interpreter, would just add value and increase the quality of their health care. Wow. Think of that. (Participant 4)

I think just having a face, socially, a face to relate to, to know, breaks down a barrier and just gives a bit more immediacy and intimacy. (Participant 3)

\section{Perceived Challenges Associated with Implementation of Telemedicine}

Study participants raised two central concerns about delivering health care over an electronic medium: financial compensation and sacrificing patient interaction, especially at the primary-care level. There was no disagreement amongst participants. Again, financial compensation is related to navigating a complex billing system: 
The problem you would run into again and again is the compensation piece ... This would be an issue unless you were willing to find a network of specialists who are willing to take one call a week, or something like that, and not worry about payment. (Participant 2)

A challenge is billing, because our medical-legal system has not been designed to be in favour of indirect consultation. (Participant 1)

That would be awesome ... if it was possible. If it was possible not only for the refugees but for all of [my patients], I would love to be able to talk to a specialist ... by videoconferencing or something and show them a skin condition and ask them what they think. It takes too much time or the specialist won't like doing that. You have to send them a referral and do it properly. (Participant 5)

Sacrificing patient interaction, perhaps the most deleterious consequence of implementing telemedicine services, was a central concern of study participants. The clinician-patient relationship is unique in that health-care providers rely upon the verbal tone and/or body language of the patient during diagnosis and treatment. Likewise, the patient places his or her general health in the hands of the clinician. In light of this delicate balance, the clinician must be a skilled communicator capable of understanding the complexities of this in-person interaction:

The biggest downfall, potentially, would be that the clients don't engage with that kind of way of communicating with a clinician. (Participant 4)

The other thing is, working with kids, I can't imagine trying to interact with them over telehealth or trying to do a developmental assessment without being able to play with them-I just can't see doing that over telehealth ... I've heard while chatting with other colleagues who also conduct mental health work ... that when they use telephone translation services versus in-person translation, they find that they miss a lot with the telephone because of lack of facial expressions and body language. Many different cultural groups place a lot of meaning in this. (Participant 1)

The biggest downfall, potentially, would be that the clients don't engage with that kind of way of communicating with a clinician. (Participant 4)

\section{Scoping Literature Review}

Twenty-seven articles satisfied inclusion criteria and were selected for review, with the literature review complementing interview data and increasing the credibility of the conclusions. Given the nature of a scoping literature review, research findings have been organized into six sorting categories based on the article title and core content: (1) telemedicine uptake; (2) telepsychiatry; (3) telemonitoring; (4) telephone assistance; (5) telecollaboration; and (6) mobile health services. Only those sections most relevant to the discussion have been elaborated upon; the full data-set is available upon request from the first author.

\section{Telemedicine Uptake}

Seven articles focused on telemedicine uptake, that is, usage patterns of a specific telemedicine service or a network of services in a geographic area or within a specific patient database. All articles focused on providing vulnerable and/or marginalized populations with increased access to health-care services.

Remote and at-home health-screening methods surpass issues of socio-economic status, gender, 33 occupation, 34 and geographic location, and have the potential to provide culturally specific services to a target population. ${ }^{35}$ Electronic screening methods provide an immediate link between patients and health-care providers and was the focus of five articles.

In highlighting the first study, videoconferencing-based clinics for rural, Native Americans allowed health-care professionals to offer culturally specific mental-health services to this population..$^{6}$ Across socio-demographic indicators such as race, ethnicity, socio-economic status, age, and sex, Kontos et al. conclude that young female patients of higher socio-economic status are more likely to utilize ehealth services. ${ }^{37}$ Reifels et al. report increased usage of applied psychological services, i.e., an electronic mental health service spanning telephone consultation or web-based cognitive behavioural therapy, despite certain patients preferring face-to-face visits..$^{8}$ With a similar conclusion, Gabrielian et al. report that while most patients were satisfied with an in-home messaging service, some participants preferred the in-person rapport. ${ }^{39}$ In contrast, and in light of the rapid flow of information between parties, Gagnon et al. conclude that technology will not negatively influence the physician-patient relationship. ${ }^{40}$ In either case, concern about straining the physician-patient relationship was raised by interview participants, and the results suggest that this issue may be overcome if the patient is interacting with a specialist physician while in the same room as his or her primary-care provider. ${ }^{41}$

Finally, and perhaps most relevant to this research article, Schulz et al. studied the first 120 consultations provided at a refugee telehealth clinic in Australia. This clinic allows a general practitioner to sit with the patient while video-conferencing with a specialist physician who is in a distant geographic location. However, the authors note that the viability of this clinic is entirely dependent upon 
continued Medicare funding provided by the Australian government. ${ }^{2}$

In the seventh article, Hitt et al. assessed the usage of a novel gynecological screening technique, telecolposcopy. As it is not applicable to this research article, a more thorough discussion can be found at the corresponding reference. ${ }^{43}$

\section{Telepsychiatry}

Six articles focused on the delivery of psychiatric services over an electronic medium. The focus of four articles was the adaptation of psychiatric services to deliver culturally competent care, that is, providing the patient with a psychiatrist, over an electronic medium, who is of a similar ethnic origin and/or able to speak the native language of the patient. As this was a central concern of interview participants, each article necessitates a brief mention. Yeung et al. discuss the provision of telepsychiatric services for Chinese immigrants in nursing homes. While the service was efficient and improved access to health-care services for the population, the authors note that participants were provided with an initial face-to-face consultation before a subsequent virtual visit. ${ }^{44}$ Mucic reports an international telepsychiatric service between Denmark and Sweden for asylum-seekers, refugees, and migrants. Patients report that the lack of physical contact between patient and physician was compensated by cultural similarities. 45 In a similar study, the authors provided a comparable service and, despite increased patient satisfaction, several participants expressed issues of confidentiality of information. ${ }^{46} \mathrm{Next}$, a telepsychiatric service for Korean immigrants connected patients to a culturally competent health-care professional. 47 In this study, unexpected technical issues, such as poor audio-visual connection, posed a barrier in care, with the authors reiterating the need for a community-based partnered approach.

Further discussion on two additional studies exploring the efficacy of electronic psychiatric services in areas of conflict 48 and using "avatar therapy" 49 can be found at the corresponding references.

\section{Telemonitoring}

Telemonitoring includes the study of diagnostic and selfmonitoring equipment and was the focus of two articles. While telemonitoring equipment is usually designed to empower the patient to actively participate in his or her medical treatment, the potential cost of this equipment, at the expense of the health-care provider, poses a serious challenge. ${ }^{\circ}$ Further, Terschüren, Mensing, and Mekel have shown that while patients may be receptive to such equipment, acceptance generally declines with increasing age. ${ }^{51}$

\section{Telephone Assistance}

Telephone assistance refers to providing medical support or monitoring for patients over a landline or a mobile phone. Eight articles focused on telephone assistance for vulnerable populations. Telephone counselling has provided emotional support for patients, ${ }^{52}$ improved treatment timeline, 53 increased medication adherence, 54 and provided early screening opportunities for pain management. 55

\section{Telecollaboration}

Telecollaboration, or the ability to work with colleagues and patients in various geographic areas, in real time, was the focus of one article..$^{56}$

\section{Mobile Health Services}

Mobile health services refer to portable health-care services such as audio-video equipment, mobile health clinics, and mobile monitoring systems. Three articles were found within this sorting category. 57

\section{Discussion and Conclusion}

The objective of this study was twofold: first, to provide the reader with an overview of the barriers to accessing health services for refugees in Hamilton; and second, to explore the efficacy of telemedicine services in remediating such barriers, especially with respect to accessing specialist care. Research results from the interview transcripts and the scoping literature review were congruent with one another, demonstrating robust research methodology.

\section{Health Services Accessibility}

During the interview sessions, the clinicians spoke of obstacles facing refugee patients when accessing health services, all of which are supported by the current body of literature. Thus, research results can be used to contribute to the literature concerned with health services accessibility for refugee populations. The results have demonstrated that the current model of care and understanding of health, in the Canadian context, has exacerbated barriers to health services access encountered by refugee patients.

Before and after migration from the country of origin, the refugee patient is forced to interact with international aid workers, government officials, and health-care professionals, among others. From a clinician's point of view, any communication barriers will be approached using the current model of care and understanding of health, a framework that prevents many refugee patients from seeking adequate care, according to study participants. While this issue may not be unique to refugees, it is likely that the residual trauma from mental and/or physical violence will exacerbate these issues. 
The results demonstrated that financial compensation for clinicians in Hamilton (and likely elsewhere in Ontario, given similar funding policies) has deterred some clinicians from serving refugee patients. The current medical-legal landscape in Canada, and particularly the evolving IFHP for refugee patients, has created confusion among healthcare providers. The authors suggest that this confusion may project onto the patient. Should a patient wish to make an appointment with a clinician, and if care is denied, the patient may generalize anger and frustration across all health care providers.

\section{Telemedicine Services: Implementation, Utilization and Recommendations}

The results suggest that two elements are required for proper implementation of telemedicine services: a communitybased partnered approach, and specialist consultation, including compensation. Results from the interview transcripts and the scoping literature review equally advocated for a community-based partnered approach. Ye et al. call for a community-based partnered approach, which involves local ethnic community centres in designing telepsychiatric services. ${ }^{5}$ While a community-based partnered approach was not explicitly discussed in the remaining references, the implicit mention of employing health-care professionals of similar cultural and/or ethnic background similar to that of the patient implies the evolution of such a concept. On the basis of professional experience, interview participants harmoniously advocated for such a solution. It is suggested that the community-based partnered approach will remedy many of the barriers created by the current model of care and understanding of health. Further, the integration of community, in a professional context, addresses any issues a health-care provider may have in connecting with a patient's cultural background.

Specialist consultation is a continued challenge for clinicians serving refugee populations. The lack of ethnically or culturally appropriate specialist physicians in the immediate Hamilton referral network begs attention. For example, telepsychiatric services have been able to connect patients with health-care professionals whose language and cultural background is similar to that of the patient. ${ }^{9}$ Such services are especially valuable for patients with a small co-ethnic community. The interview transcripts have shown that clinicians in Hamilton desire a large referral network, including a multitude of specialist physicians who are willing to regularly communicate with the primary-care clinician and the refugee patient. The primary-care clinician serves as a buffer in understanding the medical assessment, interpreting nonverbal cues of the interaction, and bringing an additional expert medical opinion. The concept of telecollaboration, or the collaboration of health-care professionals over an electronic medium, was highlighted during interview sessions and within the literature. The interview transcripts revealed that clinicians can collaborate quite easily with specialist physicians over an electronic medium. However, issues with IFHP-associated financial compensation has prevented such interaction from becoming routine. To remedy this issue, each participant called for a change in health policy legislation to address compensation for electronic consultations. Schulz et al. have demonstrated the efficacy of a telehealth clinic specific to refugee patients in Australia. The authors note that the unique health needs of this patient database necessitates its own telehealth clinic. Further, in outlining the technical challenges and successes of such a clinic, the authors note that continued support by Australian Medicare is the lifeline for electronic consultation. ${ }^{60}$

The limitations of implementing and delivering telemedicine services are many. Sacrificing face-to-face interaction, the foundation of the clinician-patient relationship, was a recurring concern amongst interview participants and within the literature. However, this limitation applies only if the primary-care clinician is communicating with the patient over an electronic medium. On the other hand, if the primary care clinician is in the immediate vicinity of the patient while communicating with a specialist physician, this restriction no longer applies. The literature also expressed concern about confidentiality and apprehension when utilizing telemedicine services. In highlighting one study, Mucic reports that patients may not feel comfortable sharing personal health information over an electronic medium. ${ }^{61}$ Thus, health-care professionals requiring patients to share such information over video-conferencing, etc., should ensure the patient is aware of all confidentiality measures.

Overall, the expected benefits associated with the implementation of telemedicine in the health-care setting include empowering patients to participate in their own continuity of care, ${ }^{62}$ increased medication adherence, ${ }^{63}$ and offering specialized health-care services across geographic boundaries. ${ }^{64}$ Finally, the integration of technology into a community-based partnered approach provides the patient with access to culturally and ethnically sensitive health care services-a factor that is likely to increase patient satisfaction and overall compliance.

To maintain transparency, the limitations of this research study must be addressed. First, a small sample size necessitates a discussion of sampling bias. The subset population who volunteered to participate in this study, given that they frequently work with refugees, may hold opinions that are similar to or different from the remainder of the medical community. Purposive, non-probability sampling of study participants was utilized to minimize sampling bias. While 
we targeted a relatively small group of health-care professionals, we continued sampling until a reasonably similar opinion was offered by all health-care professionals. While we cannot explicitly say data saturation was achieved, we believe that, given the already small number of professionals who satisfy inclusion criteria and that many community professionals do not work with refugees, saturation was achieved, although further replication of this work, including expanding the number of interviewees, is needed.

Two future research objectives are recommended. First, a call for further adaptation of the medical-legal landscape concerning the utilization of telemedicine services within and across Canadian provinces. ${ }^{65}$ In combination with IFHP-associated confusion, issues of providing health-care services across provincial or national boundaries further exacerbate such matters. The authors of this study recommend that further research must be conducted within this field for several reasons: first, to provide unimpeded and rapid access to primary and specialist health-care services for refugee populations; second, to promote the integration of telemedicine services into such care; and third, to educate health-care professionals on the updated standards of care. We recommend either interviews with services users, to explore patient perceptions of such services, or an intervention study comparing telemedicine services to the current standard of care.

Second, and perhaps most important, the core concepts of a community-based partnered approach must be integrated into routine telemedicine practice, especially when accessing specialist services. At its core, this approach relies upon trust in a health-care professional who is willing to engage in ethnically and culturally appropriate conversations with the patient. This may also be accomplished using a combination of resettlement services and/or a specialized primary care centre tailored to serving refugee populations.

\section{Glossary}

Telemedicine: the provision of health care using specialized technology. This may include telephone services, electronic video consultation, remote consultation following image acquisition, etc. Telemedicine is frequently referred to as telehealth.

Telemedicine uptake: monitoring usage patterns of a specific telemedicine service or a network of services in a geographic area or within a specific patient database.

Telepsychiatry: the provision of psychiatric services over an electronic medium. The inherent personable nature of psychiatry frequently involves electronic video consultation.

Telemonitoring: the study of diagnostic and self-monitoring equipment, usually designed to empower patients to actively participate in their health care. Telemonitoring does not include the study of consumer usage patterns.

Telephone assistance: the provision of medical assistance/ advice to patients over the telephone.

Telecollaboration: the ability to collaborate with colleagues, in real time, over an electronic medium, such as video-conferencing.

Mobile health services: portable health-care services such as audio-video equipment, mobile health clinics, and mobile monitoring systems.

\section{Notes}

1 Jennifer Asanin and Kathi Wilson, "I Spent Nine Years Looking for a Doctor': Exploring Access to Health Care among Immigrants in Mississauga, Ontario, Canada," Social Science \& Medicine 66, no. 6 (March 2008): 1271-83.

2 Marie McKeary and Bruce Newbold, "Barriers to Care: The Challenges for Canadian Refugees and Their Health Care Providers," Journal of Refugee Studies 23, no. 4 (2010): 523-45.

3 Chandni Joshi, Grant Russell, I-Hao Cheng, Margaret Kay, Kevin Pottie, Margaret Alston, Mitchell Smith, Bibiana Chan, Shiva Vasi, Winston Lo, Sayed Shukrullah Wahidi and Mark F. Harris, "A Narrative Synthesis of the Impact of Primary Health Care Delivery Models for Refugees in Resettlement Countries on Access, Quality and Coordination," International Journal for Equity in Health 12, no. 1 (2013): 1-14.

4 Panos Vostanis, "Meeting the Mental Health Needs of Refugees and Asylum-seekers," British Journal of Psychiatry 204 (2014): 176-77.

5 Joshi et al., "Narrative Synthesis."

6 Vostanis, "Meeting the Mental Health Needs."

7 Christine Carrasco, Melanie Gillespie, and Monika Goodluck, "Accessing Primary Care in Canada: Giving Voice to the Perceptions and Experiences of Racialized Immigrants," In Ethnic and Pluralism Studies Conference, 2-22, 2009.

8 Ibid.

9 Joshi et al., "Narrative Synthesis."

10 Ibid.

11 Emily J. Hadgkiss and Andre M.N. Renzaho, "The Physical Health Status, Service Utilisation and Barriers to Accessing Care for Asylum-seekers Residing in the Community: A Systematic Review of the Literature," Australian Health Review 38 (2014): 142-59.

12 Canada, Federal Government of Canada, The Canada Health Act: Overview and Options (Ottawa: Minister of Justice, 2005).

13 Government of Canada, "Interim Federal Health Program Policy,” 2014, http://www.cic.gc.ca/english/department/ laws-policy/ifhp.asp.

14 Ibid. 
15 Ibid.

16 Kevin Pottie, Ricardo Batista, Maureen Mayhew, Lorena Mota and Karen Grant, "Improving Delivery of Primary Care for Vulnerable Migrants," Canadian Family Physician 60 (2014): 32-40.

17 Jiali Ye, Ruth Shim, Tim Lukaszewski, Karen Yun, Soo Hyun Kim, and George Ruth, "Telepsychiatry Services for Korean Immigrants," Telemedicine and E-Health 18, no. 10 (2012): 797-802.

18 Albert Yeung, Daniel P. Johnson, Nhi-Ha Trinh, WanChen Claire Weng, and Joseph Kvedar, "Feasibility and Effectiveness of Telepsychiatry Services for Chinese Immigrants in a Nursing Home," Telemedicine and E-Health 15, no. 4 (2009): 336-41.

19 Stacie Anne Deslich, Timothy Thistlethwaite, and Alberto Coustasse, "Telepsychiatry in Correctional Facilities: Using Technology to Improve Access and Decrease Costs of Mental Health Care in Underserved Populations," Permanente Journal 17, no. 3 (2013): 80-6.

20 Ye et al., "Telepsychiatry Services."

21 Yeung et al., "Feasibility and Effectiveness of Telepsychiatry Services."

22 Deslich, Thistlethwaite, and Coustasse, "Telepsychiatry in Correctional Facilities."

23 Government of Ontario, "Get Medical Advice: Telehealth Ontario," 2015, https://www.ontario.ca/page/ get-medical-advice-telehealth-ontario.

24 Ontario Telemedicine Network (отN), "отN: About Us," 2015, https://otn.ca/en.

25 Government of Ontario, "Get Medical Advice."

26 Ontario Telemedicine Network, "отN."

27 Ibid.

28 Y. S. Lincoln, S. A. Lynham and E. G. Guba, "Paradigmatic Controversies, Contradictions, and Emerging Confluences, Revisited," in The Sage Handbook of Qualitative Research, ed. N. K. Denzin and Y. S. Lincoln, 4th ed., 97-128 (Thousand Oaks, CA: Sage, 2011).

29 K. Charmaz, "Grounded Theory," in Approaches to Qualitative Research: A Reader on Theory and Practice, ed. S. N. Hesse-Biber and P. Leavy, 496-521 (New York: Oxford University Press, 2003); Charmaz, "Grounded Theory in Methods in Social Justice Research," in Denzin and Lincoln, Sage Handbook of Qualitative Research, 359-8o.

30 Marcel Dijkers, "What Is a Scoping Review?" KT UPdate 4, no. 1 (2011): 1-5.

31 Hilary Arksey and Lisa O'Malley, "Scoping Studies: Towards a Methodological Framework," International Journal of Social Research Methodology: Theory \& Practice 8, no. 1 (2005): 19-32.

32 Ibid.

33 Emily Kontos, Kelly D. Blake, Wen-Ying Sylvia Chou, and Abby Prestin, "Predictors of eHealth Usage: Insights on the Digital Divide from the Health Information National Trends Survey 2012," Journal of Medical Internet Research 16, no. 7 (2014): 1-16.
34 Sonya Gabrielian, Anita Yuan, Ronald M. Andersen, James McGuire, Lisa Rubenstein, Negar Sapir, and Lillian Gelberg, "Chronic Disease Management for Recently Homeless Veterans: A Clinical Practice Improvement Program to Apply Home Telehealth Technology to a Vulnerable Population," Medical Care 51, no. 3 (2013): 44-51.

35 Jay Shore, L. Jeanne Kaufmann, Elizabeth Brooks, Byron Bair, Nancy Dailey, W. J. "Buck” Richardson, James Floyd, Jeff Lowe, Herbert Nagamoto, Robert Phares, and Spero Manson, "Review of American Indian Veteran Telemental Health," Telemedicine and E-Health 18, no. 2 (2012): 87-94. 36 Ibid.

37 Kontos et al., "Predictors of eHealth Usage."

38 Lennart Reifels, Bridget Bassilios, Kylie E. King, Justine R. Fletcher, Grant Blashki, and Jane E. Pirkis, "Innovations in Primary Mental Healthcare," Australian Health Review 37 (2013): 312-17.

39 Gabrielian et al., "Chronic Disease Management for Recently Homeless Veterans."

40 Marie-Pierre Gagnon, Lise Lamothe, Marilynne Hebert, Jacques Chanliau, and Jean-Paul Fortin, "Telehomecare for Vulnerable Populations: The Evaluation of New Models of Care," Telemedicine and E-Health 12, no. 3 (2006): 324-31.

41 Ibid.

42 T. R. Schulz, M. Richards, H. Gasko, J. Lohrey, M. E. Hibbert, and B.-A. Biggs, "Telehealth: Experience of the First 120 Consultations Delivered from a New Refugee Telehealth Clinic," Internal Medicine Journal 44, no. 10 (2014): 981-5.

43 Wilbur C. Hitt, Gordon Low, Tommy Mac Bird, and Rachel Ott, "Telemedical Cervical Cancer Screening to Bridge Medicaid Service Care Gap for Rural Women," Telemedicine and E-Health 19, no. 5 (2013): 403-8.

44 Yeung et al., "Feasibility and Effectiveness of Telepsychiatry Services."

45 Davor Mucic, "International Telepsychiatry: A Study of Patient Acceptability," Journal of Telemedicine and Telecare 14 (2008): 241-3.

46 Davor Mucic, "Transcultural Telepsychiatry and Its Impact on Patient Satisfaction," Journal of Telemedicine and Telecare 16 (2010): 237-42.

47 Ye et al., "Telepsychiatry Services."

48 Hussam Jefee-Bahloul, "Use of Telepsychiatry in Areas of Conflict: The Syrian Refugee Crisis as an Example," Journal of Telemedicine and Telecare 20, no. 3 (2014): 167-8.

49 Debra M. Quackenbush and Aaron Krasner, "Avatar Therapy: Where Technology, Symbols, Culture, and Connection Collide," Journal of Psychiatric Practice 18, no. 6 (2012): 451-9.

50 Paul Y. Takahashi, Gregory J. Hanson, Jennifer L. Pecina, Robert J. Stroebel, Rajeev Chaudhry, Nilay D. Shah, and James M. Naessens, "A Randomized Controlled Trial of Telemonitoring in Older Adults with Multiple Chronic 
Conditions: The Tele-ERA Study," BMC Health Services Research 10 (2010): 255.

51 Claudia Terschüren, Monika Mensing, and Odile $\mathrm{Cl}$ Mekel, "Is Telemonitoring an Option against Shortage of Physicians in Rural Regions? Attitude towards Telemedical Devices in the North Rhine-Westphalian Health Survey, Germany," BMC Health Services Research 12 (2012): 95-104.

52 Karen O. Anderson, Guadalupe R. Palos, Tito R. Mendoza, Charles S. Cleeland, Kai-Ping Liao, Michael J. Fisch, Araceli Garcia-Gonzalez, Alyssa G. Rieber, L. Arlene Nazario, Vicente Valero, Karin M. Hahn, Cheryl L. Person, and Richard Payne, "Automated Pain Intervention for Underserved Minority Women with Breast Cancer," Cancer 121, no. 11 (2015): 1882-9o.

53 Shannon Madore, Kristin Kilbourn, Patricia Valverde, Evelinn Borrayo, and Peter Raich, "Feasibility of a Psychosocial and Patient Navigation Intervention to Improve Access to Treatment among Underserved Breast Cancer Patients," Support Care Cancer 22, no. 8 (2014): 2085-93.

54 Neda Ratanawongsa, Vijay K. Bhandari, Margaret Handley, Thomas Rundall, Hali Hammer, and Dean Schillinger, "Primary Care Provider Perceptions of the Effectiveness of Two Self-Management Support Programs for Vulnerable Patients with Diabetes," Journal of Diabetes Science and Technology 6, no. 1 (2012): 116-24.

55 Jenni Burt, Richard Hooper, and Lynda Jessopp, "The Relationship between Use of NHs Direct and Deprivation in Southeast London: An Ecological Analysis," Journal of Public Health Medicine 25, no. 2 (2003): 174-6; Allison J. Applebaum, Katherine N. DuHamel, Gary Winkel, Christine Rini, Paul B. Greene, Catherine E. Mosher, and William H. Redd, "Therapeutic Alliance in TelephoneAdministered Cognitive-Behavioral Therapy for Hematopoietic Stem Cell Transplant Survivors," Journal of Consulting and Clinical Psychology 80, no. 5 (2012): 811-16; Joanne Shaw, Phyllis Butow, Ming Sze, Jane Young, and David Goldstein, "Reducing Disparity in Outcomes for Immigrants with Cancer: A Qualitative Assessment of the Feasibility and Acceptability of a Culturally Targeted Telephone-Based Supportive Care Intervention," Supportive Care in Cancer 21 (2013): 2297-2301; Matthew Price, Deborah Williamson, Romina McCandless, Martina Mueller, Mathew Gregoski, Brenda Brunner-Jackson, Eveline Treiber, Lydia Davidson, and Frank Treiber, "Hispanic Migrant Farm Workers' Attitudes toward Mobile Phone-Based Telehealth for Management of Chronic Health Conditions," Journal of Medical Internet Research
15, no. 4 (2013): 1-8; Courtney R. Lyles, Dean Schillinger, Andrea Lopez, Margaret Handley, Neda Ratanawongsa, and Urmimala Sarkar, "Safety Events during an Automated Telephone Self-Management Support Intervention," Journal of Diabetes Science and Technology 7, no. 3 (2013): 596-601.

56 Charles Safran, "The Collaborative Edge: Patient Empowerment for Vulnerable Populations," International Journal of Medical Informatics 69 (2003): 185-90.

57 Ehimen Aneni, Ingrid H. De Beer, Laura Hanson, Bas Rijnen, Alana T. Brenan, and Frank G. Feeley, "Mobile Primary Healthcare Services and Health Outcomes of Children in Rural Namibia," Rural and Remote Health 13 (2013): 2380-93; David W. Callway, Christopher R. Peabody, Ari Hoffman, Elizabeth Cote, Seth Moulton, Amado Alejandro Baez, and Larry Nathanson, "Disaster Mobile Health Technology: Lessons from Haiti," Prehospital and Disaster Medicine 27, no. 2 (2012): 148-52; T. Gardner, P. Gavaza, P. Meade, and D. M. Adkins, "Delivering Free Healthcare to Rural Central Appalachia Population: The Case of the Health Wagon," Rural and Remote Health 12 (2012): 1-10.

58 Ye et al., "Telepsychiatry Services."

59 Yeung et al., "Feasibility and Effectiveness of Telepsychiatry Services."

6o Schulz et al., "Telehealth."

61 Mucic, "Transcultural Telepsychiatry."

62 Burt, Hooper, and Jessopp, "The Relationship between Use of NHs Direct and Deprivation in Southeast London."

63 Madore et al., "Feasibility of a Psychosocial and Patient Navigation Intervention."

64 Ratanawongsa et al., "Primary Care Provider Perceptions"; Shaw et al., "Reducing Disparity in Outcomes for Immigrants with Cancer."

65 B. M. Dickens and R. J. Cook, "Legal and Ethical Issues in Telemedicine and Robotics," International Journal of Gynecology and Obstetrics 94 (2006): 73-8.

Anthony Robert Sandre is a medical student of the Michael G. DeGroote School of Medicine, Faculty of Health Sciences, McMaster University, Hamilton, Ontario. The author may be contacted at anthony.sandre@medportal.ca.

K. Bruce Newbold is a professor of geography and the director of the School of Geography and Earth Sciences, Faculty of Science, McMaster University, Hamilton, Ontario. The author may be contacted at newbold@mcmaster.ca. 